\title{
1 \\ Mobile Computing: A Research Perspective
}

\author{
Pravin Bhagwat Satish K. Tripathi \\ \{ pravin, tripathi\}@cs.umd.edu \\ Dept of Computer Science \\ University of Maryland \\ College Park, MD 20770
}

\section{Introduction}

The recent advances in wireless communication technology, coupled with the progress in hardware miniaturization, are taking us into a new era of computing, popularly known as nomadic computing. Battery powered, untethered computers are likely to become a pervasive part of our computing infrastructure [9]. There are, however, many technical challenges involved before the vision of ubiquitous computing can be realized. Paramount among these is the challenge of providing continuous, location independent network access to mobile computers. Traditionally, data networking protocols have been designed based on the assumption that network nodes are stationary. With the emergence of laptop computers this assumption no longer holds. From a network architecture point of view, a wireless network is another subnet attached to the backbone network. The key difference with respect to wired networks, however, lies in the mobility of hosts enabled by wireless access. Host mobility affects design considerations of communication systems at all layers of protocol stack. To name a few:

- At the physical layer, channel characteristics vary with the location of the user, and, because of mobility, vary in time. A mobile radio link is hindered by a number of propagation mechanisms, namely, multipath scattering from objects near the mobile transmitter, shadowing by dominant obstacles, and attenuation mechanisms on the propagation path between transmitter and receiver.

- At the data link layer, channel coding is to be chosen in accordance with the specific character of the fading radio channel. In contrast to wired channels with errors randomly distributed in time, bursts errors are experienced in mobile channels. A typical mobile channel allows relatively reliable communication during certain periods, interrupted by other periods of particularly poor communication known as fades.

- At the network layer, the network access point of a host may change from time to time as the user moves from one network to another. Networks must be capable of tracking the location of mobile hosts, and be able to route packets to mobile hosts at all time.

- At the transport layer, new mechanisms are needed so that active transport layer connections do not break as mobile hosts switch network access points. Wireless links coupled with host motion violate some of the basic design assumptions around which existing Transport Layer 
protocols were architected. For example, losses in wireless links are mistakenly interpreted by the transport protocol as the signs of congestion, and the corrective action taken by the transport protocol reduces the efficiency of the wireless link.

- At the application layer, new operating system primitives are required which provide support for disconnected and autonomous operation in face of intermittent network connectivity. Existing applications which depend on network support typically 'hang' when network connectivity is disrupted. Examples of such applications include NFS, X-windows and numerous other client-server applications.

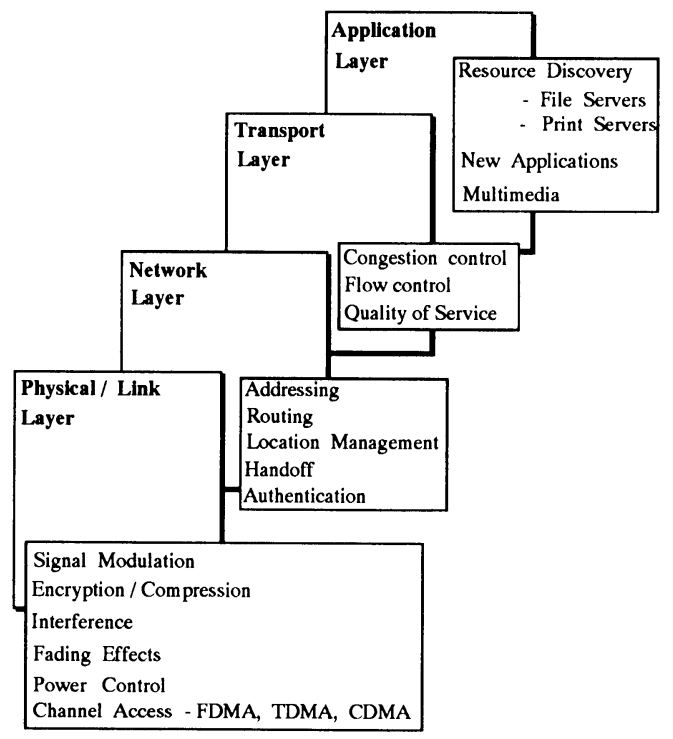

Figure 1: Mobility and the Layered Protocol Design

The issues summarized above capture the essence of all current research activity in the area of future generation mobile data communication systems. New system design constraints posed by host mobility require a careful re-evaluation of protocol design considerations at each layer in the protocol stack. In this paper, however, we limit our discussion to the impact of mobility on the design of the network and the transport layer protocols. First we present a discussion of the mobility problem from the Internet routing perspective, pointing out what makes this problem difficult to solve in practice. The next section is devoted to a short survey of all existing mobile-ip solutions. In section 4.1, we point out deficiencies in the existing transport protocols. Finally, we conclude with a short note on how host mobility affects some of the application design considerations. 


\section{Network Layer and Mobility}

In this section we explore various network layer concepts that are of importance in the design of any mobile networking system. We also explore how these pertain to mobile systems, and what additional features are needed.

\subsection{Internet Addressing and Routing}

The Internet is a large collection of networks which share the same address space and inter-operate using a common set of protocols, such as TCP/IP [13, 14]. The Internet provides connection-less best effort delivery service to all users. Data are carried in the form of NPDUs ${ }^{1}$ which contain source and destination addresses. Routers inspect destination address and forward NPDUs on to appropriate link based on the information contained in the routing table. The following three definitions will help clarify the subsequent discussion:

- Name: is a location independent identifier of a host. E.g. 'mimsy' is the name of the mailserver in out dept.

- Address: indicates where a given host is located. E.g. mimsy's address 128.8.128.8 indicates that it is connected to network 128.8.128

- Route: tells how to get to a destination

The mapping between name and address is maintained in a distributed data structure, which is know as Domain Name System (DNS). Finding the address of a host is essentially a directory lookup operation. In any large size network, addresses are allocated in a topologically significant manner. This has two advantages: First, it helps reduce the size of the routing tables maintained at various routes; second, the route to reach any host can be deduced from its address.

\subsection{The Mobility Problem}

In networks where hosts are static, name to address binding never changes. Host mobility makes this binding a function of time. Therefore, the solution to the mobility problem lies in constructing a directory lookup service, which, given a host name as input, would return the current address of the host. All network applications, prior to connection setup, acquire name to address binding through DNS lookup and then use the address of the peer entity for the entire duration of the connection. If name to address binding changes during an active transport session (as would be the case if the peer host moves) then some mechanisms are also required to notify affected hosts of this change.

Unfortunately, providing a solution for host mobility in the existing Internet environment is not so simple. The difficulty of providing a good solution stems from many sources. Internet has already grown over half a billion hosts in population, which makes any changes to host software almost impossible to achieve. Any changes to routing infrastructure, too, are not easy to achieve since routers in different addressing domains are managed by different administrative authorities. Difficulties are not always administrative in nature. There are some technical problems too. Over

\footnotetext{
${ }^{1}$ Network Protocol Data Unit
} 
the years the Internet has evolved around the assumption that hosts are stationary. This assumption manifests itself in the following two forms:

1. Domain Name System has no provision to handle dynamic address changes.

2. Names and Addresses are used synonymously. E.g., Transport protocols such as TCP [14], and UDP [12] use host address as host identifier.

The second problem is more critical, and it is the sole reason which makes mobility a difficult problem to tackle. If it were only the first problem, someday DNS could be replaced by a newer version which would efficiently handle dynamic address changes. The second constraint requires that host addresses be preserved, regardless of a host's point to attachment to the network. Its implication is that the address of a mobile host bears no topological significance. As an immediate consequence, the existing routing infrastructure cannot route packets to mobile hosts. A solution might be to keep per-mobile-host routing information at all routers, but this completely breaks the hierarchical model of routing, causing the size of routing tables to grow unbounded. Thus, the problem of supporting mobile hosts within Internet is not just keeping track of where hosts are, rather, in addition, it has to do with designing a mechanism for packet forwarding to mobile hosts without modifying and sacrificing the scalable nature of the Internet routing mechanism.

\subsection{The Solution Approach}

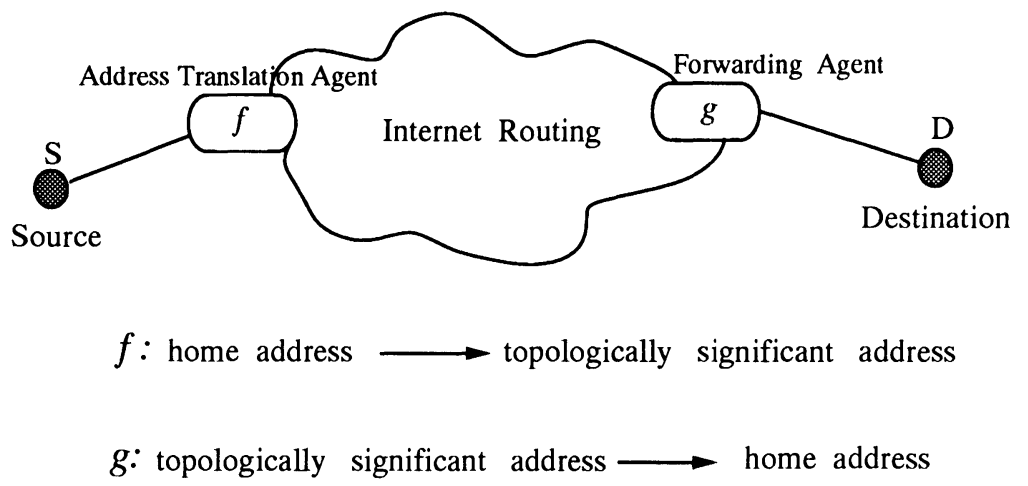

Figure 2: Packet Forwarding Model

The home address of a mobile host ceases to be topologically significant once it is not connected to its home subnet. An elegant solution can be designed by associating a forwarding address with each mobile host. A forwarding address is always topologically significant, and, therefore, it can be used within the existing routing system for the purpose of delivering packets to mobile hosts. Usually, the forwarding address is associated with a specialized entity known as a forwarding agent. This agent must be capable of directly delivering packets to mobile hosts in its domain. The agent which maintains mapping between a mobile host and its associated forwarding agent is called an address translation agent. 
With the inclusion of address translation agents and forwarding agents, the operation of packet forwarding can be easily illustrated. Refer to figure 2 which illustrates how packets from a stationary host $(S)$ are routed to a mobile host $(D) . S$ sends out packets which are addressed to the home address of $D$. These are intercepted by an address translation agent $(f)$ which readdresses these packets towards the forwarding agent $(g)$. Once these packets arrive at the forwarding agent, it strips readdressing information and delivers these packets to the mobile host.

Mobility in the Internet is essentially a re-addressing problem. Encapsulation and Source Routing are two ways of constructing the re-addressing function. In Encapsulation method a new packet header is appended at the beginning of the original packet. The outer header contains the address of the forwarding agent while the inner header contains the home address of the mobile host. Since the Internet routing system only looks at the outer packet header, it routes this packet to the forwarding agent. Forwarding agent strips the outer packet header and delivers the inner packet locally to the mobile host. Source Routing is another technique which can be used to perform re-addressing function. Using IP's source routing option, an address translation agent can cause packets addressed to a mobile host's home address to be routed via a forwarding agent.

A mobile host is always associated with some packet forwarding agent ${ }^{2}$. This association needs to be maintained, and updated at the address translation agent each time a mobile host moves from the coverage area of one forwarding agent to another. The protocol used for managing this information is called the location update protocol. All solutions proposed for the Internet mobility problem differ in terms of their choice of where to locate these agents, what the location update protocol is, and whether to use encapsulation or source routing. On surface these solutions may appear different, but they all conform to the model outlined above.

\section{Mobile-IP}

Mobile-IP is a generic term used to refer to all proposals aimed at solving the mobility problem in the Internet. Over the last two years several proposals, including one from us, have been made to address this problem $[5,1,18,19,16]$. Following is a brief outline of approach used in each one of them.

Columbia Scheme The scheme proposed by Ioannidis[5,6] relies on a group of cooperating Mobile Support Routers, which advertise reachability to the same (sub)net. Each mobile host, regardless of its location within a campus, is always reachable via one of the Mobile Support Routers (MSR). When a host sends a packet to a mobile host, it first gets delivered to the MSR closest to the source host. This MSR encapsulates the packet and delivers it to the target MSR which strips the encapsulation header and relays the original packet to the mobile host. This approach is optimized to work within a campus environment and cannot be easily extended to support wide area mobility.

Sony Scheme In Sony's proposal $[18,17]$, a mobile host is assigned a new temporary address when it is attached to a new network. The mapping between the home address and the temporary address of a mobile host is kept in an Address Mapping Table (AMT), which is maintained at the routers. Packets transmitted to the home address of the mobile host get intercepted by some router

\footnotetext{
${ }^{2}$ Usually a wireless base-station functions as a packet forwarding agent
} 
which holds an AMT entry for the mobile host. An address conversion is performed by the router before the packets are forwarded to the physical location of the mobile host. This method requires modifications to routers and host software and has problems inter-operating with the existing hosts unless so-called 'conversion gateways' are used.

MITL Scheme Another proposal to support mobile hosts is from Matsushita[19]. This method is also based on the encapsulation approach. A mobile host is assigned a temporary address when it visits a new network. The packets destined to the home address of the mobile host are intercepted by a Packet Forwarding Server(PFS). The PFS encapsulates the packet and forwards it using the temporary address of the target mobile host. The problem with this method is that routing is always sub-optimal unless the software on all stationary hosts is modified.

LSR Scheme The LSR approach $[1,11]$ is based on the use of an existing IP option and therefore, does not require any changes to the existing hosts and routers. The key idea is that each packet originating from a mobile host contains enough routing information that can be used by the remote host to send reply back to the source along an optimal path. The key feature of our design is that all functionality needed to manage and process location information is embedded in specialized entities, called Mobile Router and Mobile Access Station. This allows seamless integration of mobile hosts into current networking infrastructure without requiring any changes to existing routers and hosts. A summary of the notable features of our design is as follows:

- The system inter-operates with any TCP/IP compliant system.

- It achieves optimal routing.

- The system enables mobile hosts to roam without causing any disruption in active transport layer sessions.

- No modifications are required to routers and non-mobile hosts.

- Mobile hosts retain their address regardless of their location. This allows all networking applications to run on mobile hosts without any modifications.

- Support for mobility is provided completely within the network layer. This makes our solution very general and independent of any hardware technology used for wireless communication.

An implementation of the LSR scheme currently exists under AIX and MACH in our wireless lab facility at Maryland. The current implementation, in addition to providing mobile to mobile communication capability over wireless infrared link, also allows mobiles to move from the domain of one base-station to another without breaking any active transport layer connections.

\section{Transport Layer Issues}

\subsection{Effect of wireless link and mobility on the Transport Layer}

The network architecture providing ubiquitous access to mobile users will consist of a high speed broadband backbone and a set of wireless networks attached to the periphery. Solutions proposed 
for Mobile-IP would ensure continuous delivery of packets to mobile hosts even as they move from the range of one base-station to another. Enabling the packet forwarding function, as provided by any Mobile-IP system, would make a wireless system functional. However, the design of such a system would not be complete without a careful evaluation of how existing transport protocols and applications would perform in such an environment.

The integration of wireless and broadband networks will give rise to connections with routes encompassing both networks. Existing transport protocols, such as TCP and TP4 have been optimized to operate over wired links, where packet losses are rare and bandwidth is plenty. Wireless segments on the other hand are bandwidth limited and have error characteristics which are often bursty in nature. Moreover, user mobility can cause further packet losses during handoff [3]. It is evident that the underlying assumptions that guided the design of existing transport layer protocols are violated, and, as a result, a connection that encompasses a wireless link may encounter poor and unpredictable performance. To provide good and predictable performance in an integrated wireless and wired network, the transport layer issues need to be revisited. For any solution to be acceptable, it is important to provide inter-operability with the existing population of wired hosts, since they constitute the majority of the infrastructure. The challenge, therefore, is to architect changes in such a way that re-engineered protocols operate in the new environment with little or no changes to the existing infrastructure.

In the following we take a closer look at some of problems outlined above. The discussion is centered around dynamics of TCP, as it is the most widely used transport protocol in the Internet. Similar problems, however, are expected to arise in context of other protocols as well.

\subsection{TCP's reaction to packet losses}

TCP is designed around the assumption that links are reliable. It interprets packet losses to be resulting from congestion and consequently reacts by reducing its congestion window down to one and entering into slow-start $[7,15]$ phase. This control action is justified if underlying links are reliable in which case the true reason behind the packet loss is most likely to be congestion and the only remedy is to reduce input rate. However, triggering a congestion avoidance mechanism in response to each packet loss in wireless medium may lead to unacceptable level of performance in some cases [10]. Infact, if wireless link is indeed flaky then TCP's slow start mechanism may repeatedly get triggered, resulting in a a scenario where TCP's window size stays at the minimum possible value.

The source of this problem lies in inability to detect whether the losses are due to congestion or they are due to burst errors in the wireless link. Better control mechanisms can be designed if some form of feedback is available from wireless hardware about the quality of wireless link. Unfortunately, current generation wireless adapters do not have any provision to provide this information.

Another plausible solution to this problem is to use a reliable link layer protocol over the wireless segment. The intuition behind why this solution would work is that a reliable link layer would substantially reduce the effective packet loss rate see by the transport layer. This approach too does not warrant good performance due to reasons outlined in the next section; primary reason being the complex interaction of the link layer retransmission mechanism with the dynamics of the round trip time(RTT) and retransmission timeout(RTO) computation of TCP [4]. 


\subsection{Problems originating from RTO computation}

For each connection, TCP maintains a running average of RTT (referred to as $\tau_{r t t}$ ). RTT is computed by measuring the time difference between the instant the packet was released in the network and the moment ACK for this packet arrives back at the source. In addition to $\tau_{\text {rtt }}$, TCP also keeps a running average of variance in RTT (referred to as $\delta_{r t t}$ ). Finally, the retransmission timeout(RTO) (i.e., the period beyond which a packet is retransmitted if its acknowledgement is not received) is computed as follows:

$$
\begin{gathered}
\tau^{i+1}=\alpha \tau^{i}+(1-\alpha) R T T \\
\delta^{i+1}=\beta \delta^{i}+(1-\beta)\left|\tau^{i+1}-R T T\right| \\
R T O^{i}=\tau^{i}+2 \delta^{i}
\end{gathered}
$$

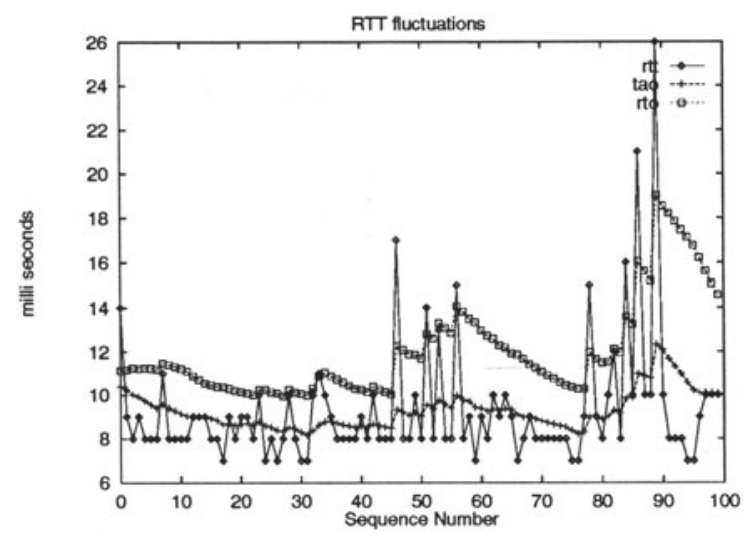

Figure 3: Round Trip Time and Retransmission Timeout Computation in TCP

Figure 3 shows a sample of TCP's $\tau_{r t t}$ and RTO computation. The data shown in the graph is taken from actual round trip time measurements collected over 3 hop Ethernet.

If delay variance in the wired segment is relatively small (as indeed would be the case if the backbone an ATM network), and losses in the wireless segment are bursty then it is easy to see that adding more reliability in the link layer segment may not always help. When a connection is in low error state, RTO would converge to the actual RTT of the session. Any packet loss in the wireless segment would not only trigger retransmission at the link layer, but it will also cause TCP's retransmission timer to expire resulting in another retransmission from the source This not only renders the retransmission at the link layer useless but it also wastes bandwidth of the wireless medium.

Burst errors in wireless medium may also adversely affect Karn's RTO adjustment algorithm [8]. According to Karn's algorithm, ACKs for retransmitted packets are never used for updating RTO 
estimate, rather RTO estimate is doubled each time TCP retransmits a packet ${ }^{3}$. If multiple retransmissions of a packet fail (which is very likely when the wireless link is in burst error mode) then the RTO estimate climbs at an exponential rate. As a result, even after the wireless link returns to a normal state, TCP does not transmit any packet until its timer expires. This behavior causes long periods of inactivity on part of transport protocol, resulting in substantial throughput degradation.

\section{Summary}

The rapid emergence of mobile computers as a popular, and increasingly powerful, computing device is presenting new challenges to the research community. A great deal of research is being carried out to support the needs of the growing mobile computer industry. A key challenge is to build the underlying infrastructure to support wireless communication. In this system, the main issue is not so much that the communication medium is wireless, but rather that the system must deliver information in the face of a constantly changing environment precisely because the end devices are on move. Intergrating this system with the existing wired infrastructure, applications and services is another important design consideration. The networking research attempts to solve this problem by providing a uniform location-independent networking interface to the mobile devices so that the end-users can use their desktop applications on their laptops without any modifications or reconfiguration. Most application designers, however, take a different approach. Their solution relies on making applications location- and situation-aware [2]. The idea here is to build enough intelligence into applications so that they can make use of the locally available resources to dynamically configure themselves in a distributed fashion. The issues discussed in this paper are only a few points in the vast territory of open problems that yet remain to be explored. Research in this area is only beginning, and it will take many more years of research effort before we develop a full understanding of all design tradeoffs.

\section{References}

[1] Pravin Bhagwat and Charles Perkins. A Mobile Networking System based on Internet Protocol(IP). In Proceedings of USENIX Symposium on Mobile and Location Independent Computing, pages 69-82, Cambridge, MA, Aug 1993.

[2] Pravin Bhagwat, Charles Perkins, and Satish K. Tripathi. Transparent resource discovery for mobile computers. In Workshop on Mobile Computing Systems and Applications, Dec 1994.

[3] Ramon Caceres and Liviu Lftode. The effects of mobility on reliable transport protocols. In Proceedings of the International Conference on Distribnuted Computing Systems, pages 12-20, Poznan, Poland, 1994. IEEE.

[4] Antonio DeSimone, Mooi Choo Chuah, and On-Ching Yue. Throughput performance of transport-layer protocols over wireless lans. In Proceedings of the Conference on Global Communications (GLOBECOM), pages 542-549. IEEE, 1993.

\footnotetext{
${ }^{3}$ Reference [8] provides a detailed rationale behind why this approach has been adopted in TCP
} 
[5] John Ioannidis, Dan Duchamp, and Gerald Q. Maguire Jr. IP-based Protocols for Mobile Internetworking. In Proceedings of ACM SIGCOMM, pages 235-245, 1991.

[6] John Ioannidis and Gerald Q. Maguire Jr. The Design and Implementation of a Mobile Internetworking Architecture. In Proceedings of Winter USENIX, pages 491-502, San Diego, CA, Jan 1993.

[7] Van Jacobson. Congestion avoidance and control. ACM Computer Communication Review, 18(4):314-329, August 1988. Proceedings of the Sigcomm ' 88 Symposium in Stanford, CA, August, 1988.

[8] Phil Karn and Craig Partridge. Improving round trip time estimates in reliable transport protocols. ACM Transactions on Computer Systems, 9:364-373, November 1991.

[9] Randy H. Katz. Adaptation and Mobility in Wireless Information Systems. IEEE Personal Communication Magazine, 1(1):6-17, Feb 1994.

[10] T. V. Laksman and U. Madhow. Performance Analysis of window-based flow control using TCP/IP: the effect of high bandwidth-delay products and random loss. In High Performance Networking, 1994.

[11] Charles Perkins and Pravin Bhagwat. A Mobile Networking System based on Internet Protocol. IEEE Personal Communication Magazine, 1(1):32-41, Feb 1994.

[12] J. Postel. User Datagram Protocol. RFC 768, Aug 1980.

[13] J. Postel. Internet Protocol. RFC 791, Sep 1981.

[14] J. Postel. Transmission Control Protocol. RFC 793, Sep 1981.

[15] Scott Shenker, Lixia Zhang, and Dave Clark. Some observations on the dynamics of a congestion control algorithm. ACM Computer Communication Review, pages 30-39, October 1990.

[16] Bill Simpson. draft-ietf-mobileip-protocol-03.txt. Draft RFC - work in progress, May 1994.

[17] Fumio Teraoka and Mario Tokoro. Host Migration Transparency in IP Networks. Computer Communication Review, pages 45-65, Jan 1993.

[18] Fumio Teraoka, Yasuhiko Yokote, and Mario Tokoro. A Network Architecture Providing Host Migration Transparency. In Proceeding of ACM SIGCOMM, Sept 1991.

[19] Hiromi Wada, Takashi Yozawa, Tatsuya Ohnishi, and Yasunori Tanaka. Mobile Computing Environment Based on Internet Packet Forwarding. In proceeding of Winter USENIX, pages 503-517, San Diego, CA, Jan 1993. 